\title{
Quality indicators for primary care mental health services
}

\author{
T Shield, S Campbell, A Rogers, A Worrall, C Chew-Graham, L Gask
}

Qual Saf Health Care 2003;12:100-106

See editorial commentary, pp 85-6

See end of article for authors' affiliations

Correspondence to: Dr S Campbell, National Primary Care Research and Development Centre, University of Manchester, Williamson Building, Oxford Road, Manchester M13 9PL, UK;

stephen.campbell@man.ac.uk

Accepted for publication 14 October 2002

\begin{abstract}
Objectives: To identify a generic set of face valid quality indicators for primary care mental health services which reflect a multi-stakeholder perspective and can be used for facilitating quality improvement.

Design: Modified two-round postal Delphi questionnaire.

Setting: Geographical spread across Great Britain.

Participants: One hundred and fifteen panellists representing 11 different stakeholder groups within primary care mental health services (clinical psychologist, health and social care commissioner, community psychiatric nurse, counsellor, general practitioner, practice nurse/district nurse/health visitor, psychiatrist, social worker, carer, patient and voluntary organisations).

Main outcome measures: Face validity (median rating of 8 or 9 on a nine point scale with agreement by all panels) for assessing quality of care.

Results: A maximum of 334 indicators were rated by panels in the second round; $26 \%$ were rated valid by all panels. These indicators were categorised into 21 aspects of care, 11 relating to general practices and 10 relating to health authorities or primary care groups/trusts. There was variation in the total number of indicators rated valid across the different panels. Overall, GPs rated the lowest number of indicators as valid $(41 \%, n=138)$ and carers rated the highest number valid $(91 \%, n=304)$.

Conclusions: The quality indicators represent consensus among key stakeholder groups in defining quality of care within primary care mental health services. These indicators could provide a guide for primary care organisations embarking on quality improvement initiatives in mental health care when addressing national targets and standards relating to primary care set out in the National Service Framework for Mental Health for England. Although many of the indicators relate to parochial issues in UK service delivery, the methodology used in the development of the indicators could be applied in other settings to produce locally relevant indicators.
\end{abstract}

1 he central role played by primary care in the recognition and delivery of care for people with mental health problems is now widely acknowledged. ${ }^{1}$ However, ensuring the quality of primary mental health care remains a challenge in both developed and developing nations and, even in countries such as the UK with well established primary care and mental health services, there is considerable geographical variation in the quality of care delivered.

In England it is only recently that a National Service Framework and strategy for mental health care has been developed. In the past, whether and how mental health care should be subjected to quality assessment was interpreted and implemented by those working in different localities. Moves towards establishing a standardised and generic system of quality measurement in mental health care in England began in the 1990s when the Clinical Standards Advisory Group focused on both the management of schizophrenia ${ }^{2}$ and depression $^{3}$ and, with the latter, moved towards examining the quality of provision in primary as well as secondary care. This was subsequently followed by the National Service Framework for Mental Health ${ }^{4}$ which included the role of primary care in the delivery of mental health care (box 1). The implied development of protocols within primary care as part of Standard 2 of the National Service Framework indicates the very low baseline from which issues of quality within primary care mental health services start.

The English National Service Framework identifies primary care groups and primary care trusts as the lead organisations for developing roles and responsibility for primary care and access to services. ${ }^{5}$ They are required to address methods of quality improvement in primary mental health care. However,
Box 1 National Service Framework for Mental Health for England

The National Service Framework for Mental Health forms part of the Government's agenda to drive up quality and reduce unacceptable variations in health and social services. Developed by health and social care professionals, service users and carers, health and social service managers and partner agencies, the National Service Framework for Mental Health focuses on the mental health needs of working age adults up to 65. The document sets out national standards, national service models, local action and national underpinning programmes for implementation over a 10 year period. In addition, the National Service Framework for Mental Health provides a series of national milestones and performance indicators to assure progress and to support effective performance management. An organisation framework for providing integrated services and commissioning services across the spectrum of mental health service provision is also included.

Standard 1: Mental health promotion

Standards 2 \& 3: Primary care and access to services Standards 4 \& 5: Effective services for people with severe mental illness

Standard 6: Caring about carers

Standard 7: Preventing suicide

there are few validated measures of mental health care available for use. Valid indicators of mental health care are important in assessing and improving quality of care as they can 
Box 2 Eleven single specialty panels involved in the Delphi process

- Eight professional panels

- clinical psychologist

- health and social care commissioner

- community psychiatric nurse

- counsellor

- general practitioner

- nurse (practice, district, health visitor)

- psychiatrist

- social work

- Carer panel

- Patient panel

- Voluntary organisation panel

show variations in care, including suboptimal care. ${ }^{6}$ They can also be used as a catalyst for facilitating quality improvement initiatives, which is particularly relevant given the difficulties and challenges faced by primary care groups and primary care trusts in implementing the mental health National Service Framework. ${ }^{7}$ Although there are a number of guidelines relating to mental health problems, these tend to focus on specific conditions and care provided within secondary care. There is little relevant internationally published research in this area, but in the US a National Inventory of Mental Health Quality Measures identified a number of gaps between existing measures for mental health care and quality related needs of primary stakeholders. ${ }^{8}$

Thus, there are few indicators available for quality assessment of primary mental health care, and few that can be applied at the system level-for example, practice or primary care organisation-rather than at the level of the diagnostic group (such as depression or anxiety) or that reflect the views of key stakeholders in the primary mental health setting, particularly patients and carers.

We present the results of a Delphi survey ${ }^{9-11}$ which aimed to develop a generic set of face valid service quality indicators for primary care mental health services that reflect the perspectives of key stakeholder groups. Although the Delphi approach has been used previously within mental health care, this has largely been to set research priorities, ${ }^{12}$ establish models of good practice, $^{1314}$ and identify essential components of various aspects of mental health care. ${ }^{15}{ }^{16}$ Previous work has not been concerned with developing measures for quality improvement within mental health care and has generally included only a limited number of perspectives. Indeed, the patient's perspective has been conspicuously absent from consensus methods in the past ${ }^{17}$ Given that the acceptability of indicators depends on their perceived relevance and value to potential users, ${ }^{18}$ the inclusion of different perspectives-including those of patients and carers-is an important part of the process.

\section{METHODS}

\section{Process}

A two-round postal Delphi survey was carried out between March and July 2000. The study was conducted entirely by post for cost purposes and to include more respondents over a greater geographical area.

\section{Panellists}

Eleven single specialty panels were convened. These consisted of eight separate professional panels, one carer panel, one patient panel, and one made up of people from voluntary organisations (box 2). Panellists from professional groups reflected a combination of practitioners and those selected on the basis of their national reputation-that is, publication record, involvement in specialist societies, or as nominees of professional organisations. Similarly, the patient and carer panels contained patients and carers as well as known

\section{Box 3 Definition of terms}

\section{Validity}

Extent to which the indicator related to an aspect of care which is important for providing high quality care for mental health problems in primary care.

\section{Clarity}

Extent to which the indicator was expressed in clear, precise, and unambiguous language.

\section{Agreement}

Round $1: 60 \%$ or more of ratings within a panel fell within the region 7-9

Round 2: $75 \%$ or more of ratings within a panel fell within the region 7-9.

patient/carer advocates and those involved in key patient/carer organisations. The composition of panels reflected a geographical spread across Great Britain. Each panel was composed of 9-12 representatives. Panellists were sent a letter of invitation to participate. Those who were unable to take part were asked to nominate a possible replacement.

\section{Indicators}

A preliminary set of indicators was constructed from a number of sources. Firstly, previously published guideline statements and quality indicators and standards relating to primary mental health care were reviewed, as well as sources of published/grey literature produced by key organisations. Secondly, patient focus groups were convened to identify aspects of care (and thus indicators) considered important from the patient/user perspective. Finally, 22 of the standards set out in the National Service Framework for Mental Health for England were included. A total of 367 indicators were identified which covered a range of aspects of care at practice, primary care group, and health authority levels.

\section{Questionnaire}

The list of potential indicators was developed into a Delphi questionnaire which is reproduced in full on the journal website (www.qshc.com). The indicators were categorised into three levels of service which related to those provided by individual practices, primary care groups, and health authorities. Campbell and colleagues ${ }^{19}$ have proposed that quality of care is a combination of access (whether users get the care they need) and effectiveness of clinical and interpersonal care (whether the care is effective when they get it). Practice level indicators were categorised according to these two elements. Some of the indicators could have been placed in one or more categories but, to avoid duplication, these indicators were put into only one category.

Subsequent amendments were made following consultation with a six member reference group composed of a health care commissioner, general practitioner, patient, community psychiatric nurse, counsellor, and psychiatrist.

\section{Delphi round 1}

During the first round the Delphi panellists were asked to rate the indicators on two continuous integer 9-point scales for validity and clarity (box 3 ) with $1=$ lowest and $9=$ highest rating.

Indicators were clarified and modified after feedback from the first round according to written comments made by panellists. Fifteen indicators receiving an overall median clarity score across panels of $\leqslant 6$ were rewritten but retained; 38 indicators were discarded on this basis because of perceived duplication and lack of relevance to primary care, while an additional five indicators were included. A maximum number of 334 indicators could therefore potentially have been 
Box 4 Practice level indicators

\section{Practice: ACCESS}

\section{Access}

- Patients are able to make a routine appointment to see a GP within 2 days

- There is equity of access to talking treatments regardless of ethnic origin, age, place of residence, socioeconomic status, and sex

- There is good access to integrated and community based mental health services out of hours (deputising/cooperative/GP and community mental health services)

- A member of the primary health care team is available as a point of contact for all patients to talk to in an emergency

Practice policies and procedures

- There is a written protocol/strategy to ensure that specific difficult to place groups are not excluded from registration with the practice (e.g. homeless, drug misusers, residential care residents)

- There is an agreed definition of severe and enduring mental illness which is explicit and standard within the practice

- There is a written complaints procedure which is prominently displayed

- Practices have a written policy for dealing with violent or abusive patients

- Clear written practice protocols are in place for obtaining specialist help in an emergency/crisis situation

Information for patients and carers

- Patients are given information about their condition, treatments, medication (including side effects) and coping strategies

- Information (i.e. practice information leaflets, health promotion leaflets) is easy to understand and available in appropriate languages for patients and carers whose first language is not English

Medical records

- Medical records, including computerised records, are up to date and summarised

- The confidentiality of medical records is protected and ensured at all times

- Details of currently prescribed maintenance drugs are prominently recorded in the medical record

Practice: EFFECTIVENESS

Patient-staff relations

- Staff treat all people registered with the practice with respect, courtesy and consideration irrespective of age, sex, religious/cultural beliefs, or diagnosis

- Staff are aware that mental health problems affect people of both sexes, all ages, backgrounds, and socioeconomic status

- Staff are aware that patients with a mental illness may be concerned about feelings of stigmatisation and are treated in a way to minimise these feelings

- Staff are aware that a diagnosis of mental illness does not make an individual legally unable (incompetent) to decide about treatment

- Patients are not made to feel that they are wasting health professionals' time

- Patients are listened to and taken seriously

- Physical symptoms in people with mental health problems are taken seriously and not automatically considered as psychosomatic

- Staff are responsive to patients' fears and concerns about the unwanted side effects of medication, including addiction

- Staff are aware of the potential impact of a mental health problem on patient behaviour (i.e. non-attenders with mental health problems should be accorded greater latitude)

- Patients with a mental health condition are treated as individuals with individual needs and not as a "diagnosis" (i.e. not labelled as depressed but treated as a person with depression)

- Health professionals communicate simply in language that is easy to understand

Confidentiality and consent

- Confidential discussions take place in private

- There is an appropriate (i.e. private, quiet, relatively non-clinical) room for counselling/visiting mental health staff

- Where practicable, patient consent is sought before giving information to carers

Comprehensive assessments

- Assessment of physical, psychological, and social health needs in patients with mental health problems includes an assessment of risk

- Assessment takes into account language barriers; the needs of people with disabilities (including sensory impairment), ethnic, cultural and religious preferences

\section{Patient involvement in treatment plans}

- Patients are as fully involved as practicable in the formulation and delivery of their care (e.g. through the use of self-care plans)

- Where appropriate, patients are offered treatments other than medication

- Treatment plans are individually tailored for each patient

- Patients are involved, unless impracticable, in any decisions about referral

- Where practicable, patients are informed of the reasons for referral to specialists or other professionals

Psychotropic prescribing

- Prescribing for mental health conditions is based on up to date evidence and, where available, local management protocols

- Choice of medication is based on individual patient factors including the desirability of sedation, previous response to a drug treatment including adverse reactions, co-morbid psychiatric or medical conditions, concurrent drug treatment, and relative risk of medication in overdose

- Clear and accessible information is provided with every prescribed psychiatric drug including information about the potential benefits and unwanted side effects

- Prescribed medication is at the minimum necessary dosage

- No drug is prescribed unless the health professional understands the potential efficacy and side effects

- Patients not responding to first line drug treatment at the therapeutic dosage are asked about adherence

- Patients are offered referral to a mental health worker if they are experiencing difficulties undertaking withdrawal from medication

Psychological treatment

- Counsellors, psychotherapists, and other practitioners of talking treatments working within practices:

- are appropriately trained and hold appropriate qualifications

- have regular supervision

- make the boundaries of the patient-professional relationship clear and transparent 


\section{Box 4 continued}

\section{Follow up}

- Patients with a diagnosis of mental illness are offered regular appointments to monitor and follow up treatment, symptoms, side effects and adherence

- Patients on repeat maintenance drugs are offered regular reviews of their medication including monitoring for possible side effects and interactions with other drugs

- During withdrawal from any medication patients are seen regularly for monitoring and support

included within each panel's round 2 questionnaire. However, not all panels received all 334 indicators because individual median panel scores were created for each indicator. Indicators which did not receive a median validity score of 7-9 with agreement within a panel were not included in that panel's second round questionnaire. Agreement was defined as
$60 \%$ or more scores in the top (7-9) tertile (box 3). ${ }^{20}$ Each panel therefore had a unique second round questionnaire.

\section{Delphi round 2}

Each panellist was sent a second round questionnaire which included three types of feedback based on first round validity

\section{Box 5 Higher level (primary care group/trust and health authority) indicators}

\section{Registration with a general practice}

- There is a written protocol/strategy to ensure that specific difficult to place groups are not excluded from registration with general practices (e.g. homeless, drug misusers, residential care residents)

\section{Equity of access}

- Services (including talking treatments) are available to all practices

- There are written policies on equity of access to services

- Patients are able to make a routine appointment to see a GP within 2 days

- There is evidence of a clear local programme to address and combat discrimination and to promote social inclusion of individuals and groups with mental health problems (e.g. through collaborative initiatives and partnerships with other agencies)

- The frequency of use of translator services by practices is monitored and is linked to practice population profiles

Referrals

- Written guidelines are in place to ensure that, where services are not provided locally, GPs can refer patients outside their locality

- There is a single point of rapid access for urgent referrals to specialist mental health services (duty professional or fast tracking service)

\section{Psychotropic prescribing}

- There are agreed written protocols and guidelines, based on best available evidence, for prescribing psychotropic medication

- There are locally agreed written protocols for prescribing across the primary-secondary care interface including hospital initiated prescribing

\section{Comprehensive range of good quality mental health services}

- There is a designated individual who acts as the mental health lead

- Specialist services are based on locally agreed written service plans and agreements which include the range, quality, and volume of mental health services

- A range of services is available, including talking treatments

Mental health promotion

- Procedures exist which demonstrate a commitment to promoting the mental health of all individuals and communities

- Health authorities or primary care trusts, in collaboration with social services and primary care groups, work with individuals and communities to promote mental health

Out of hours care

- There is good access to integrated and community based mental health services out of hours (deputising/cooperative/GP and community mental health services)

- There are locally agreed written standards and protocols for the delivery of out of hours care for mental health problems

- There are locally agreed standards and protocols for responding to mental health crises out of hours

Training and development

- There is a demonstrable commitment to promote continuous professional and practice development in primary care

- Practices are offered protected time for GPs and nurses to attend appropriate training courses

- There is evidence that training is organised to address specific training needs, for example:

- mental health key workers and the Care Programme Approach

- GPs and the Mental Health Act (1983)

- health visitors and the management of postnatal depression

- initiatives to make all health professionals aware of the impact of a mental health problem on patients' perspectives and actions

- There are adequate numbers of approved Section 12, Mental Health Act (1983) doctors

- There is evidence of initiatives to recruit doctors trained under Section 12 of the Mental Health Act (1983)

Effective partnerships between health authorities, local authorities and primary care groups/trusts

- There is a range of collaborative initiatives in place with other key agencies demonstrating effective partnerships (local authorities, voluntary agencies, other primary care groups/trusts)

Monitoring

- There are written protocols and mechanisms in place for monitoring prescribing of psychotropic drugs

- There is a written protocol/strategy to monitor whether specific difficult to place groups are excluded from registration with general practices (homeless, drug misusers, residential care residents)

- There is evidence of monitoring to ensure that out of hours standards are met 
Table 1 Percentage of indicators rated valid by each panel after the Delphi process

Percentage of indicators rated valid after Delphi process

\begin{tabular}{lll} 
& \multicolumn{2}{l}{$\begin{array}{l}\text { Percentage of indicators rated valid } \\
\text { after Delphi process }\end{array}$} \\
\cline { 2 - 3 } Panel & $\begin{array}{l}\text { Delphi round } 1 \\
(n=367)\end{array}$ & $\begin{array}{c}\text { Delphi round } 2 \\
(n=334)^{*}\end{array}$ \\
\hline Carer & $83 \%(n=305)$ & $91 \%(n=304)$ \\
Patient & $78 \%(n=286)$ & $86 \%(n=287)$ \\
Voluntary organisations & $79 \%(n=290)$ & $86 \%(n=286)$ \\
Community psychiatric nurse & $78 \%(n=288)$ & $86 \%(n=286)$ \\
Clinical psychologist & $76 \%(n=278)$ & $79 \%(n=263)$ \\
Health and social care & $61 \%(n=225)$ & $66 \%(n=220)$ \\
commissioner & $66 \%(n=243)$ & $66 \%(n=219)$ \\
Nurse & $64 \%(n=236)$ & $62 \%(n=206)$ \\
Social worker & $59 \%(n=216)$ & $56 \%(n=187)$ \\
Counsellor & $53 \%(n=195)$ & $49 \%(n=162)$ \\
Psychiatrist & $50 \%(n=183)$ & $41 \%(n=138)$ \\
General practitioner & &
\end{tabular}

* $n$ is given as 334 rather than 367 as only a maximum number of 334 indicators could potentially have been included in each panel's unique round 2 questionnaire.

scores for each indicator (panellist's own previous score, panellist's median panel score, and the frequency distribution of scores within the panellist's own panel). Panellists were then asked to re-rate the indicators specific to their panel for validity.

\section{Proposed analysis}

Previous research has shown that use of a higher cut off point $(\geqslant 8)$ is associated with greater reproducibility and reliability. ${ }^{21}$ Validity after second round ratings was therefore defined as an overall median for each panel of $\geqslant 8$. A more stringent level of agreement of $75 \%$ or more in the top (7-9) tertile was applied (box 3). Indicators rated with an overall validity score of 8 or 9 with agreement by all panels were considered face valid indicators. Those rated valid by all panels were operationalised into aspects of care by the research team to produce indicators which could be used pragmatically to facilitate quality improvement. $\chi^{2}$ tests were used to determine whether there were significant differences between the types of indicators rated valid by different panels-for example, between the different levels of service.

\section{RESULTS}

\section{Response rate}

A response rate of $90 \%$ was achieved in round 1 and $89 \%$ in round 2. The number of returned questionnaires on which analysis was based ranged from a minimum of eight (counsellor, community psychiatric nurse, nurse, psychiatrist) to a maximum of 11 (clinical psychologist, voluntary organisations) per panel. All final analyses reported in this paper are based on second round results.

\section{Consensus among panel ratings}

Most of the indicators $(97 \%, \mathrm{n}=324)$ were rated valid by at least one panel in round $2 ; 26 \%$ of the indicators were rated valid by all panels. The latter set of indicators represents a consensus among key stakeholder groups in defining quality of mental health care in primary care and, as such, formed the focus of the study. These indicators have been categorised into 21 aspects of care, 11 relating to general practice and 10 relating to health authorities or primary care groups/trusts (boxes 4 and 5).

\section{Differences in panel ratings}

The extent of intra-panel disagreement was extremely low with less than $1 \%$ disagreement within any one panel. Disagreement was defined as 30\% or more scores in both the bottom (1-3) and top (6-9) tertiles. ${ }^{20}$ However, there were major differences between panel ratings. Table 1 shows the variation in the total number of indicators rated valid across different panels after the first and second rounds of the Delphi process. Overall, GPs rated the lowest number of indicators as valid $(41 \%)$ and carers rated the highest number valid $(91 \%)$.

Panels rated different aspects of care as valid-for example, the GP panel did not rate protocols or guidelines for the management of separate mental health problems (including depression and anxiety) as central to providing good quality care, whereas the carer, clinical psychologist, community psychiatric nurse, patient, and voluntary organisation panels did. Moreover, $\chi^{2}$ tests showed that GPs were the only stakeholder group who significantly rated more indicators as valid at the practice level than at the level of the primary care group and health authority $(p<0.01)$. For all other groups there was either no significant difference in their ratings between different levels (commissioners, counsellors, patients, psychiatrists and social workers) or they rated significantly more indicators as important at the higher level $(\mathrm{p}<0.05)$ (carers, clinical psychologists, community psychiatric nurses, nurses, voluntary organisations). Given that the focus of this paper is the indicators which were rated valid by all panels, a more detailed account of the major differences between panel ratings will be reported separately as part of a follow up study exploring the factors which influence the ratings of stakeholders in a Delphi survey.

\section{DISCUSSION}

Our results show that it is possible to identify agreement between groups of stakeholders in identifying a generic set of valid quality indicators for primary care mental health services. All panels agreed that $26 \%$ of the indicators were valid measures of quality of care. However, the results of the Delphi survey also show that different stakeholders value different aspects of quality of care. The final set of indicators listed in boxes 4 and 5 provide a starting point for the development of quality improvement tools.

\section{Limitations of the study}

Although the Delphi process is a well validated method for assessing opinion, ${ }^{9-11}$ this study has some important limitations. Firstly, the indicators rated were based on best available evidence in 2000 and, as such, must be reviewed in the light of major new research findings. The final set of indicators cannot be considered a comprehensive assessment of service level mental health services. Rather, they represent a consensus among key stakeholder groups in defining quality of care within primary care mental health services.

Secondly, appropriate composition of panels in consensus methodologies is a key factor in determining the legitimacy of the findings. ${ }^{2122}$ Considerable care was taken to select panels in order to reflect a range of expertise. This involved combining practitioners and those with a national reputation (based on publication record and membership of national committees/organisations) for the professional groups, and also including known patient and carer advocates/members of key organisations within the patient and carer panels. However, panels could not be said to be representative of their stakeholder perspective, but merely suggestive of their profession/group.

Thirdly, the aim of the study was to identify indicators rated valid by all stakeholders/panels but, because we attempted to identify all relevant stakeholder groups, there was a built-in bias in having a larger number of professional panels than a single patient and carer panel. Even though the rating process was democratic with each panel's ratings carrying equal weight, because the carer and patient panels rated more of the indicators valid than, for example, the GP or psychiatrist panels, this meant that the final set of indicators included fewer of the aspects of care of value to patients or carers than of value 
to GPs or psychiatrists. Those panels rating the least number of indicators valid (GPs, psychiatrists, counsellors) therefore had greater influence on the final set of indicators than those rating the highest numbers of indicators valid (carers, patients, voluntary organisations, and community psychiatric nurses). However, this did not detract from the aim of the study to identify a common set of indicators.

Finally, the set of indicators developed required additional editing by the research team in order to operationalise them for use within quality improvement strategies. However, the meaning of the indicators remained intact.

\section{Implications for quality improvement}

The fact that only $26 \%$ of the quality indicators were agreed by all panels has implications for quality improvement and assurance purposes, given that the relative proportion of statements agreed amongst panels-particularly between professionals and users-differed. Such differences in the ratings of the various panels reflect the natural diversity of opinion within mental health care. The indicators rated valid by each panel also provide opportunities for quality improvement strategies which address the aspects of care rated valid by each different perspective (these are available from the authors). Policy makers in all countries intending to use consensus methods to provide a multidisciplinary approach to quality assessment and improvement within health and social care need to give thought to this issue in order to create a more equal partnership between what consumers value and what providers value in terms of quality care.

Quality indicators are defined as a measurable element of performance for which there is evidence or consensus that they can be used to assess the quality, and hence change in the quality of care provided.$^{23}$ While the indicators listed in boxes 4 and 5 represent consensus among the 11 panels, many are professional aspirations and standards rather than measurable indicators. This is perhaps a reflection of the underdeveloped and nascent evidence base from which to develop outcome based indicators for primary mental health care, and the difficulty in collecting such data in the setting of primary care even in a system as complex and well developed as that in the UK. Measurement depends on reliable data systems which, although readily available in the US health maintenance organisations, are only now in development in healthcare settings in other western countries. It is necessary to set up these systems and infrastructure before quality assurance/ control can be both feasible and reliable. We have therefore not attempted to distinguish between the conventional (measurable) quality indicators and the more aspirational indicators. While a number of the indicators will be easily measurable, others-such as those included within patient-staff relations-will require more innovative and eclectic approaches to measurement. Although this will provide considerable challenges to primary care organisations in facilitating quality improvement, the state of the literature relating quality of care to outcome in mental health care compared with conditions such as coronary heart disease ${ }^{7}$ necessitates such an approach to quality improvement.

The final set of indicators can be used for both quality improvement (which does not need measurement) and quality assessment (which does). Importantly, these indicators are not intended to provide a comprehensive tool set for measuring quality of care. Rather, they can be used as a starting point for discussion by primary care organisations and practices and as a means of assisting primary care organisations to address the national targets and standards relating to primary care set out in the National Service Framework for Mental Health for England. They also represent a potential for identifying the current baseline of care provision. In this way the indicators raise awareness around important issues of primary mental health care. This is particularly relevant in localities where

\section{Key messages}

- Valid quality indicators can show variations in the quality of care and can act as catalysts for facilitating quality improvement initiatives.

- Few indicators are available for use in primary mental health care, especially at the level of the system (practice or primary care organisation) rather than the diagnostic group, or reflecting the views of key stakeholders (patients and carers).

- A maximum of 334 indicators were rated by 11 stakeholder panels in the second round of a Delphi study, $26 \%$ of which were rated valid by all panels.

- This generic set of service quality indicators represents a consensus among key stakeholder groups in defining quality of care within primary care mental health services which can be used in quality improvement strategies.

progress in developing primary care mental health services is limited and a cultural shift to increase the profile of mental health care is required.

When applying quality indicators it is important to ensure that they are done realistically and that they reflect local circumstances. $^{24}$ Some areas of the UK have a better infrastructure for primary care mental health services than others. ${ }^{7}$ We have not therefore attempted to establish standards for these indicators. Rather, primary care organisations or practices may adopt a standard which is relevant to their local circumstances and needs and initiates debate at a local level.

Other potential uses of the indicators are to act as sign posts in defining quality of care and to indicate areas where additional resources may be required. The indicators could then be used to aid primary care organisations and/or practices to set locally achievable goals and to develop local standards of care. Given that the indicators themselves are not a comprehensive set of quality measurement tools, it may be necessary-depending on the aims of the quality improvement strategy - to supplement these indicators with other key local and national documents within this field.

\section{Conclusion}

The quality indicators developed in this study, although not comprehensive, represent a consensus among key stakeholder groups in defining quality of care within primary care mental health services. They can be used as a starting point in quality improvement strategies and as a means for assisting primary care organisations to address the national targets and standards relating to primary care set out in the National Service Framework for Mental Health for England. Although many of the indicators relate to parochial issues in UK service delivery, the methodology used in their development—with its multi-stakeholder input and involvement of patients, carers, and professionals-could be applied in other settings to produce locally relevant indicators.

\section{ACKNOWLEDGEMENTS}

The authors would like to thank the panellists who took part in the Delphi study and the following people for their contribution to the project: Martin Roland (NPCRDC, University of Manchester); Elizabeth Holford, Susannah Rix, Geoff Shepherd (Health Advisory Service); Maureen McGeorge (College Research Unit); David Morris (The Sainsbury Centre for Mental Health); and Sandra Shaw (NPCRDC). They acknowledge the contributions of the following organisations to the study: African-Caribbean Mental Health Project (Zion Community Health Resource Centre), Alcohol and Drug Services, Association of Counsellors and Psychotherapists in Primary Care (CPC), Awaaz Group, The Big Issue In The North, Birmingham Health Authority, British Association for Counselling, British Association of Social Workers, British Psychological Society (incl. BPSCORE), Carers Network Westminster, Centre for Health Services 
Research, The Central Council for Education and Training in Social Work, Chester City Primary Care Group, City of Edinburgh Council, Clinical Governance Research \& Development Unit, Clinical Standards Advisory Group, Community Psychiatric Nurse Association (CPNA), Counselling in Primary Care Trust, Drugline, Gamian Europe, Institute of Psychiatry, Kings Fund, London Borough of Sutton (Housing \& Social Services), MACA, Making Space, Manic Depression Fellowship, Mental Health Foundation, Mental Welfare Commission for Scotland, MIND, Morgannwg Local Medical Committee, The National Assembly for Wales, National Alliance of the Relatives of the Mentally Ill (NARMI), National Depression Care Training Centre, National Schizophrenia Fellowship, Primary Care Mental Health Education and Development Unit, Psychological Therapies Research Centre, Royal College of General Practitioners, Royal College of Nursing, The Sainsbury Centre for Mental Health, The Samaritans, School of Health and Related Research (ScHARR), South London and Maudsley NHS Trust, Stockport Health Authority, Stockport NHS Trust: Department of Clinical Psychology, Stockport Metropolitan Borough Council, Substance Misuse Advisory Service (SMAS), United Kingdom Advocacy Network (UKAN), United Kingdom Central Council for Nursing, Midwifery and Health Visiting, WATCH.

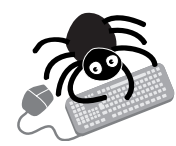

The Delphi questionnaire and accompanying glossary are available on the journal website (http://ghc.bmijournals.com/supplemental)

\section{Authors' affiliations}

T Shield, S Campbell, A Rogers, L Gask, National Primary Care Research and Development Centre, University of Manchester, Manchester M13 9PL, UK

A Worrall, College Research Unit, Royal College of Psychiatrists, London SWIH OHW, UK

C Chew-Graham, Department of General Practice, University of Manchester, Manchester M14 5NP, UK

The project was devised by LG, SC, AR and TS and managed by TS. The Delphi questionnaire was developed by SC, TS and LG. TS undertook the analyses. LG, SC, TS, CCG and AW were involved in the operationalisation of the indicators. TS, SC and LG wrote the paper with TS as the principal author. TS is the guarantor of the paper.

This project was funded out of NPCRDC core funding from the Department of Health. There are no conflicts of interest.

\section{REFERENCES}

1 Ustun TB, Sartorius N, eds. Mental illness in general health care: an international study. Chichester: John Wiley and Sons, 1995.
2 Clinical Standards Advisory Group. Schizophrenia. Volumes 1 and 2. London: HMSO, 1995

3 Clinical Standards Advisory Group. Services for people with depression. London: HMSO, 1999.

4 Department of Health. The national service framework for mental health. London: Department of Health, 1999.

5 Wilkin D, Gillam S, Leese B, eds. The national tracker survey of primary care groups and trusts: progress and challenges, 1999/2000 Manchester: University of Manchester, NPCRDC, 1999.

6 Seddon ME, Marshall MN, Campbell SM, et al. Systematic review of studies of quality of clinical care in general practice in the United Kingdom, Australia and New Zealand. Qual Health Care $2001 ; 10: 152-8$

7 Rogers A, Campbell SM, Gask L, et al. Some national service frameworks are more equal than others: implementing clinical governance for mental health in primary care groups and trusts. J Ment Health 2002;11:199-212

8 Hermann RC, Leff HC, Palmer RH, et al. Quality measures for mental health care: results from a national inventory. Med Clin Res Rev 2000;57:135-53.

9 Linstone HA, Turoff M. The Delphi survey. Method, techniques and applications. Reading, Massachusetts: Addison-Wesley, 1975.

10 Cantrill JA, Sibbald B, Buetow S. The Delphi and nominal group techniques in health services research. Int J Pharm Pract 1996;1:67-71.

11 Hasson F, Keeney S, McKenna H. Research guidelines for the Delphi survey technique. J Adv Nurs 2000;32:1008-15.

12 Wilkinson G, Williams P. Priorities for research on mental health in primary care settings. Psychol Med 1985;15:507-14.

13 Skews G, Meehan T, Hunt G, et al. Development and validation of clinical indicators for mental health nursing practice. Aust NZ Ment Health Nurs 2000;9:11-18.

14 Walker L, Barker $P$, Pearson P. The required role of the psychiatric-mental health nurse in primary health care: an augmented Delphi study. Nursing Inquiry 2000;7:91-102.

15 Fiander M, Burns T. Essential components of schizophrenia care: a Delphi approach. Acta Psychiatry Scand 1998;98:400-5.

16 Schaller G, Fournier P. Prevention priorities in mental health in Geneva: a study using the Delphi technique. Sante Publique 1998;10:191-201.

17 Kassirer JP. The quality of care and the quality of measuring it. N Engl J Med 1993;329:1263-4

18 Turpin RS, Darcy LA, Koss R, et al. A model to assess the usefulness of performance indicators. Int J Qual Health Care 1996;8:321-9.

19 Campbell SM, Roland M, Buetow SA. Defining quality of care. Soc Sci Med 2000:51:1611-25.

20 Brook RH. The RAND/UCLA appropriateness method. In: McCormick KA, Moore SR, Siegel RA, eds. Methodology perspectives. AHCPR Publication No. 95-0009. Rockville, MD: Public Health Service, US Department of Health and Human Services, 1994: 59-70.

21 Shekelle PG, Kahan JP, Park RE, et al. Assessing appropriateness by expert panels: how reliable? J Gen Intern Med 1995; Suppl 10:81.

22 Campbell SM, Cantrill JA. Consensus methods in prescribing research. $J$ Clin Pharmacol Ther $2001 ; 26: 5-14$.

23 Lawrence M, Olesen F. Indicators of quality health care. Eur J Gen Pract 1997:3:103-8.

24 Marshall M, Campbell SM. Introduction to quality assessment in general practice. In: Marshall M, Campbell SM, Hacker J, et al, eds. Quality indicators for general practice: a practical guide for health professionals and managers. London: Royal Society of Medicine, 2002: 1-6. 